\title{
Comment on "Increase in voice level and speaker comfort in lecture rooms” [J. Acoust. Soc. Am. 125, 2072-2082 (2009)] (L)
}

\author{
David Pelegrín-García ${ }^{\text {a) }}$ \\ Acoustic Technology, Department of Electrical Engineering, Technical University of Denmark, \\ Kongens Lyngby DK-2800, Denmark
}

(Received 7 October 2010; revised 13 December 2010; accepted 15 December 2010)

\begin{abstract}
Recently, a paper written by Brunskog Gade, Payá-Ballester and Reig-Calbo, "Increase in voice level and speaker comfort in lecture rooms" [J. Acoust. Soc. Am. 125, 2072-2082 (2009)] related teachers' variation in vocal intensity during lecturing to the room acoustic conditions, introducing an objective parameter called "room gain" to describe these variations. In a failed attempt to replicate the objective measurements by Brunskog et al., a simplified and improved method for the calculation of room gain is proposed, in addition with an alternative magnitude called "voice support." The measured parameters are consistent with those of other studies and are used here to build two empirical models relating the voice power levels measured by Brunskog et al., to the room gain and the voice support. (C) 2011 Acoustical Society of America. [DOI: 10.1121/1.3543940]
\end{abstract}

PACS number(s): 43.55.Hy, 43.70.Mn [NX]

Pages: $1161-1164$

\section{INTRODUCTION}

Brunskog et al., published "Increase in voice level and speaker comfort in lecture rooms" previously in this journal. ${ }^{1}$ Their work showed a possible influence of room acoustics (through a new parameter named room gain) on the vocal intensity used by teachers for talking in rooms. In addition, different subjective aspects regarding the perceived acoustic conditions while talking were studied by means of questionnaires. The work extended its relevance to the areas of ergonomics and occupational health, as it described an interaction between man and environment with possible consequences for voice health originated from working conditions. A recent epidemiological study has shown that teachers with voice problems rate classroom acoustics as an element affecting their voice much more often than those without voice problems. ${ }^{2}$ In this context, the work of Brunskog et al., could offer a reference dataset to compare the vocal performance of teachers' with and without voice problems under different acoustic conditions. However, it has been impossible to replicate the room gain measurements of Brunskog et al., in the original rooms of their study. The aim of this paper is to provide a more accurate and replicable dataset relating the voice power levels measured by Brunskog et al., to the objective parameters room gain and voice support derived with an alternative method. The first section presents the definition of room gain according to the method of Brunskog et al., pointing out some potential limitations, and it is followed by the definition of room gain and voice support according to an alternative method. The second section compares the objective measurements in the rooms of Brunskog et al., as they appear in the original study and with the alternative method. The last section describes two empirical models relating the voice power level to the room gain and the voice support.

\footnotetext{
a) Author to whom correspondence should be addressed. Electronic mail: dpg@elektro.dtu.dk
}

Note: The terms vocal intensity, voice level, and voice power level $L_{\mathrm{W}}$ are used in this paper to express the total radiated speech power from a talker. While the first term is used as a qualitative description, the other two terms are used indistinctly to express a quantitative magnitude.

\section{ROOM ACOUSTIC PARAMETERS FOR A TALKER}

Two equivalent metrics that characterize the effect of room acoustics as perceived by a talker are used: room gain $\left(G_{\mathrm{RG}}\right)$ and voice support $\left(S T_{\mathrm{V}}\right)$.

Brunskog et al., defined the room gain as the degree of amplification produced by the room on the talker's voice, as perceived by the talker himself. The calculation of room gain proposed in Brunskog et al., requires the measurement of two impulse responses (IRs) corresponding to the sound transmission path between the mouth and the ears of a dummy head: one at the room of interest $h(t)$ and another one at an anechoic chamber $h_{\text {ach }}(t)$. From these two measurements, the energy levels of the IR at the position of interest, $L_{\mathrm{E}}$, and at the anechoic room, $L_{\mathrm{E} \text {,ach }}$ are calculated

$$
\begin{gathered}
L_{\mathrm{E}}=10 \log \frac{\int_{0}^{\infty} h^{2}(t) \mathrm{d} t}{E_{0}}, \\
L_{\mathrm{E}, \mathrm{ach}}=10 \log \frac{\int_{0}^{\infty} h_{\mathrm{ach}}^{2}(t) \mathrm{d} t}{E_{0}},
\end{gathered}
$$

where $E_{0}$ is an arbitrary energy reference. The room gain is calculated as the difference between these two energy levels

$$
G_{\mathrm{RG}}=L_{\mathrm{E}}-L_{\mathrm{E}, \mathrm{ach}} .
$$

The room gain is conceptually related to Gade's objective support, ${ }^{3}$ which is widely used in stage acoustics to compare the energy of early sound reflection patterns from a music instrument to the player's ears among different rooms 
for music performance. Gade's objective support is used to characterize many different kinds of instruments, with different distances from the source to the ears of the musicians and different directivity patterns. In the case of voice, the path between mouth and ears is rather well defined.

The method of Brunskog et al. for calculating room gain is conceptually and theoretically correct and can be used to calculate the room gain at positions with very close reflecting surfaces. However, an important limitation of the method is the required measure of an IR in anechoic conditions, which can be an obstacle for many professionals. Additionally, in practice, the IR in anechoic conditions might differ from the direct sound in the measuring conditions due to changes in temperature, humidity, background noise, and distortion artifacts when measuring. The practical limitations lead to measurement error, which is illustrated in the following example.

Nine IRs in a small room, corresponding to the acoustic path between the mouth and the left ear of a Head and Torso Simulator (HATS) B\&K type 4128 (Nærum DK-2850, Denmark) with left ear simulator B\&K type 4159, were measured with the $01 \mathrm{~dB}$ Symphonie system (Limonest Cedex F-69578, France). The measurements corresponded to three repetitions at three different reproduction gains, keeping the HATS position fixed. The signal-to-noise ratio (SNR), calculated from the peak level to the noise floor level, was at least 60 $\mathrm{dB}$ in all IRs. The IRs were trimmed to the intersection of the exponential decay curve with the noise floor of the measurement with the lowest SNR (the intersection time was noted as $\left.t_{\text {min }}\right)$. The IRs were normalized to a peak amplitude of 1 , and the energy levels $L_{\mathrm{E}}$ in the interval $\left(0-t_{\mathrm{min}}\right)$ were calculated. The estimated standard deviation of $L_{\mathrm{E}}$ was $0.02 \mathrm{~dB}$, whereas the maximum difference between two measurements of $L_{\mathrm{E}}$ was $0.06 \mathrm{~dB}$. This error is not usually regarded as important, but as defined in Eq. (3), the room gain can be significantly biased by such an amount, since typical values lie between 0 and $0.6 \mathrm{~dB}$.

It would be beneficial to derive the room gain from a single IR measurement and increase the sensitivity of the method. For this, the author proposes the measurement of the IR using a HATS with a mouth simulator, according to recommendation ITU-T P.58, ${ }^{4}$ and an ear simulator with ear canal, according to recommendation ITU-T P. $57^{5}$ type 3 . The source should be at least $1 \mathrm{~m}$ away from all boundaries, including the floor, using a stand to appropriately place the HATS at the height of the head of an average standing person. The distance gap of $1 \mathrm{~m}$ allows for a time gap free of reflections of approximately $5.8 \mathrm{~ms}$. The direct sound $h_{\mathrm{d}}(t)$ is obtained by applying a window $w(t)$ to the measured IR $h(t)$ (see Fig. 1)

$$
h_{\mathrm{d}}(t)=h(t) \times w(t),
$$

where

$$
w(t)= \begin{cases}1 & t<4.5 \mathrm{~ms} \\ 0.5+0.5 \cos \left(2 \pi\left(t-t_{0}\right) / T\right) & 4.5 \mathrm{~ms}<t<5.5 \mathrm{~ms} \\ 0 & t>5.5 \mathrm{~ms}\end{cases}
$$

with $t_{0}=4.5 \mathrm{~ms}$ and $T=2 \mathrm{~ms}$. The reflected sound $h_{\mathrm{r}}(t)$ is the complementary signal

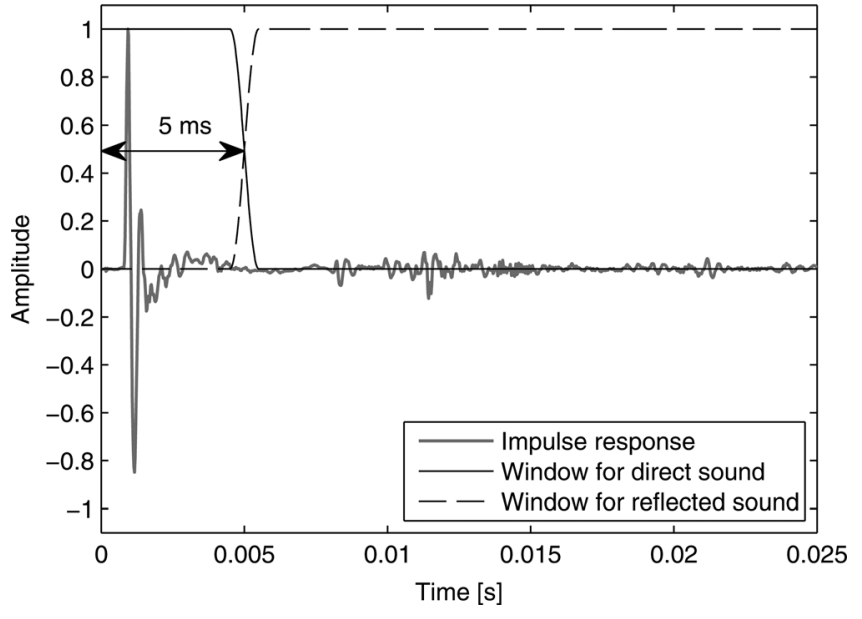

FIG. 1. Example of an IR $h(t)$ and the windowing applied to extract the direct and reflected sound.

$$
h_{\mathrm{r}}(t)=h(t) \times(1-w(t))=h(t)-h_{\mathrm{d}}(t) .
$$

The energy levels corresponding to the direct sound $\left(L_{\mathrm{E}, \mathrm{d}}\right)$ and the reflected sound $\left(L_{\mathrm{E}, \mathrm{r}}\right)$ are calculated as

$$
\begin{aligned}
& L_{\mathrm{E}, \mathrm{d}}=10 \log \frac{\int_{0}^{\infty} h_{\mathrm{d}}^{2}(t) \mathrm{d} t}{E_{0}}, \\
& L_{\mathrm{E}, \mathrm{r}}=10 \log \frac{\int_{0}^{\infty} h_{\mathrm{r}}^{2}(t) \mathrm{d} t}{E_{0}} .
\end{aligned}
$$

The voice support $S T_{\mathrm{V}}$, in analogy to Gade's objective support, is defined as the difference between the reflected sound and the direct sound from the HATS' mouth to ears IR

$$
S T_{\mathrm{V}}=L_{\mathrm{E}, \mathrm{r}}-L_{\mathrm{E}, \mathrm{d}}
$$

which is related to the room gain through the formula

$$
G_{\mathrm{RG}} \approx 10 \log \left(10^{S T_{\mathrm{V}} / 10}+1\right)
$$

This formula is obtained under the assumption that the total energy is approximately the sum of the energies corresponding to the direct and the reflected sound after windowing

$$
L_{\mathrm{E}} \approx 10 \log \left(10^{L_{\mathrm{E}, \mathrm{d}} / 10}+10^{L_{\mathrm{E}, \mathrm{r}} / 10}\right) .
$$

Gade's objective support is intended for big rooms, so the early reflections are counted from $20 \mathrm{~ms}$, and the first 10 $\mathrm{ms}$ in the IR are regarded as direct sound. This parameter cannot be used in small rooms (e.g., rooms for speech), as the early reflections are much closer to the direct sound than in large halls, and may fall in the direct sound interval or in the interval from 10 to $20 \mathrm{~ms}$, which is ignored by the definition. With the present definition of direct and reflected paths, it is possible to calculate room gain and voice support in many rooms. The only limitation is that all boundaries of the room should be $1 \mathrm{~m}$ away from the measurement equipment. 
The indirect calculation of room gain after measuring the voice support with Eq. (10) reduces the deviation in the results. Using the same IRs of the previous example, the standard deviation in the measured room gain was reduced from 0.02 to $0.004 \mathrm{~dB}$, and the maximum differences between two measurements did not exceed $0.01 \mathrm{~dB}$.

\section{ABOUT THE MEASURED PARAMETERS}

Table I shows the six rooms used in the study of Brunskog et al., with their volume and the original measurements of reverberation time $T_{30}$ and room gain, noted as $G_{\mathrm{RG}}^{\prime}$. Inspecting the original $G_{\mathrm{RG}}^{\prime}$ data, the value of $1.12 \mathrm{~dB}$ measured in the IEC 268-13 compliant listening room appears too high in comparison to that measured in the meeting room $(0.58 \mathrm{~dB})$, which is smaller and more reverberant than the IEC room. These values imply that the reflected sound pressure level in the IEC room would be about $3 \mathrm{~dB}$ higher than in the meeting room, using Eq. (10).

The room IRs in the six rooms of the study were measured again, following the procedure described in the previous section. No filtering, other than the intrinsic response of the loudspeaker, was applied to the signals for deriving the objective parameters. The values of voice support $S T_{\mathrm{V}}$ and room gain $G_{\mathrm{RG}}$, measured for each room as the average of six repetitions, are shown in Table I. The differences between old and new room gain values are indicated as $\Delta G_{\mathrm{RG}}$.

The new measurements confirm the initial suspicions. The room gain in the IEC listening room is indeed lower than in the meeting room. The room gain in the anechoic chamber was $0 \mathrm{~dB}$ in the original study by definition and it is $0.01 \mathrm{~dB}$ by the present method described here. In general, the room gain values are lower than in the original study $\left(\Delta G_{\mathrm{RG}}>0\right.$ in all cases), a fact that has been already reported. ${ }^{6}$ None of the room gain values was higher than $0.5 \mathrm{~dB}$. The voice support has a greater dynamic range and might be more suitable for use in architectural acoustics. However, in anechoic rooms, $S T_{\mathrm{V}} \rightarrow-\infty$, and the finite values measured under these conditions must be treated carefully.

\section{REVISED EMPIRICAL MODELS}

The new room gain values differ considerably from the original values. In order to enable reliable comparison with future studies, the empirical model relating voice power level from the study of Brunskog et al., to the room gain has to be recomputed. The relative voice power level $\left(\Delta L_{\mathrm{W}}\right)$ is

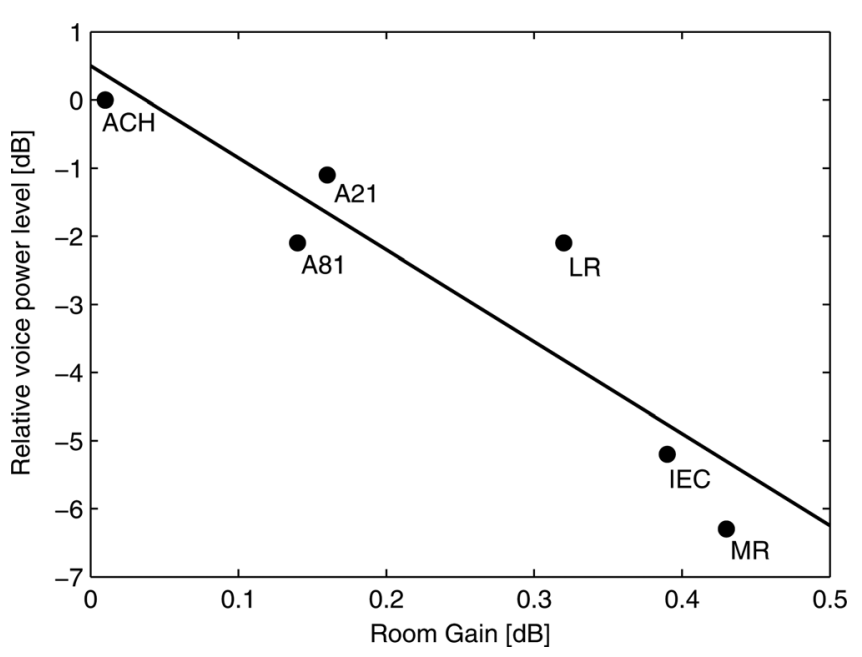

FIG. 2. Relative $L_{\mathrm{W}}$ produced by talkers in the study by Brunskog et al., as a function of the room gain. The reference $L_{\mathrm{W}}$ is the average overall $L_{\mathrm{W}}$ measured by Brunskog et al., in the anechoic chamber.

defined as the difference between the overall $L_{\mathrm{W}}$ in a certain room and the overall $L_{\mathrm{W}}$ measured in the anechoic room. A simplified linear model of only one explanatory variable is preferred

$$
\Delta L_{\mathrm{W}}[\mathrm{dB}]=0.5-13.5 \times G_{\mathrm{RG}} .
$$

The model predicts a decrease in the expected voice power level with increasing room gain $\left(R^{2}=0.83, p=0.01\right)$. Alternatively, rooms with low room gain demand higher vocal intensity from talkers. The measured values, and the regression model (12), are shown in Fig. 2. A two-variable model, similar to the one proposed in Brunskog et al., which describes the relative voice power level as a function of the room gain and the logarithm of the volume, is not significant at the $5 \%$ level $\left(R^{2}=0.83, p=0.07\right)$ and shows marginal or no influence of the logarithm of the volume on the voice levels.

Figure 3 shows the relative values of voice power level measured by Brunskog et al., versus the voice support. The critical dependence of $S T_{\mathrm{V}}$ value on the measurement SNR in the anechoic chamber suggests that voice level does not change much for very negative values of $S T_{\mathrm{V}}$, also shown with the transformed regression model using the room gain (dotted curve in Fig. 3). A linear dependence of $\Delta L_{\mathrm{W}}$ and $S T_{\mathrm{V}}$ for all the conditions studied is not a good approximation. This approximation does not exclude the possibility of modeling a linear dependence between $L_{\mathrm{W}}$ and $S T_{\mathrm{V}}$ in a limited range of

TABLE 1. Rooms in the study by Brunskog et al., and measured objective parameters. The volume $V$, reverberation time $T_{30}$, and room gain $G_{\mathrm{RG}}^{\prime}$ are taken from the paper of Brunskog et al. The room gain $G_{\mathrm{RG}}$ and voice support $S T_{\mathrm{V}}$ correspond to new measurements. The differences between old and new room gain values are indicated as $\Delta G_{\mathrm{RG}}$.

\begin{tabular}{|c|c|c|c|c|c|c|c|}
\hline Name & Abbreviation & $V\left(\mathrm{~m}^{3}\right)$ & $T_{30}(\mathrm{~s})$ & $G_{\mathrm{RG}}^{\prime}(\mathrm{dB})$ & $G_{\mathrm{RG}}(\mathrm{dB})$ & $\Delta G_{\mathrm{RG}}(\mathrm{dB})$ & $S T_{\mathrm{V}}(\mathrm{dB})$ \\
\hline Auditorium 81 & A81 & 1900 & 1.06 & 0.28 & 0.14 & 0.14 & -14.9 \\
\hline Auditorium 21 & A 21 & 1220 & 1.53 & 0.29 & 0.16 & 0.13 & -14.2 \\
\hline Lecture room 019 & LR & 190 & 0.46 & 0.42 & 0.32 & 0.10 & -11.1 \\
\hline Meeting room 112 & MR & 94 & 0.42 & 0.58 & 0.43 & 0.15 & -9.8 \\
\hline Large anechoic chamber & $\mathrm{ACH}$ & 1000 & 0.06 & 0 & 0.01 & 0.01 & -27.3 \\
\hline IEC listening room & IEC & 100 & 0.34 & 1.12 & 0.39 & 0.73 & -10.3 \\
\hline
\end{tabular}




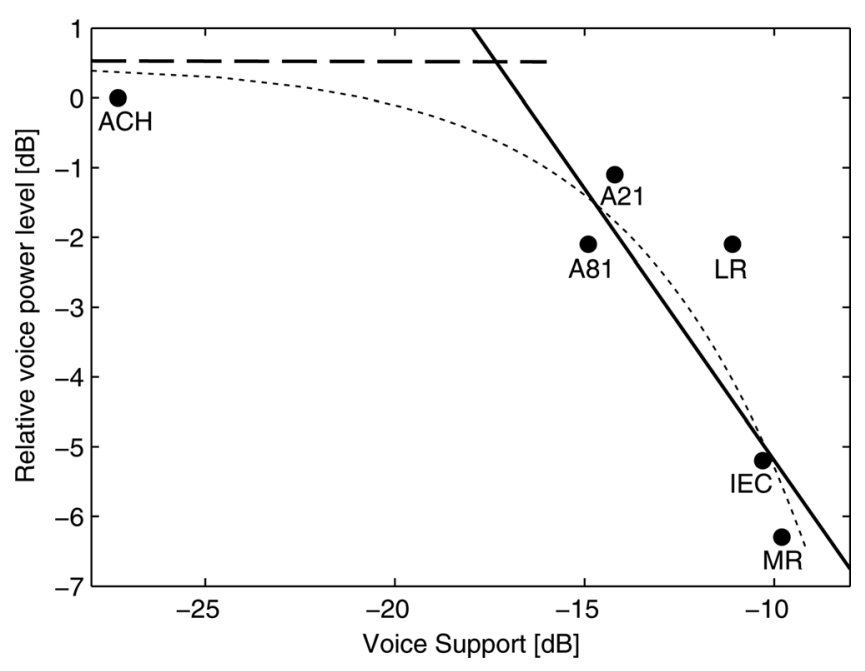

FIG. 3. Relative $L_{\mathrm{W}}$ produced by talkers in the study by Brunskog et al., as a function of the voice support. Solid line: regression model excluding the measurements in the anechoic chamber. Dashed line: expected asymptotic relative $L_{\mathrm{W}}$ value. Dotted line: regression model for room gain. The reference $L_{\mathrm{W}}$ is the average overall $L_{\mathrm{W}}$ measured by Brunskog et al., in the anechoic chamber.

$S T_{\mathrm{V}}$, as has been done in recent studies, ${ }^{7,8}$ while approaching an asymptotic $L_{\mathrm{W}}$ value for very negative $S T_{\mathrm{V}}$ (dashed line in Fig. 3). Excluding the measurement in the anechoic chamber, the best linear model (solid line in Fig. 3) is

$$
\Delta L_{\mathrm{W}}[\mathrm{dB}]=-13-0.78 \times S T_{\mathrm{V}}
$$

The accuracy of the predictions decreases with this parameter $\left(R^{2}=0.66, p=0.09\right)$. It would not be wise to conclude that the voice support is less valid than the room gain to describe the changes in voice level due to the acoustic conditions perceived by the talker. More conditions are needed to assess the robustness of room gain and voice support as explanatory variables of voice level variations due to changes in the auditory perception of one's own voice elicited by the room.

${ }^{1}$ J. Brunskog, A. Gade, G. Payá-Ballester, and L. Reig-Calbo, "Increase in voice level and speaker comfort in lecture rooms," J. Acoust. Soc. Am. 125, 2072-2082 (2009).

${ }^{2}$ V. Lyberg-Åhlander, R. Rydell, and A. Löfqvist, "Speaker's comfort in teaching environments: Voice problems in Swedish teaching staff," J. Voice (2010), available online March 26, 2010.

${ }^{3} \mathrm{~A}$. Gade, "Investigations of musicians room acoustic conditions in concert halls. Part I: Methods and laboratory experiments," Acustica 69, 193-203 (1989).

${ }^{4}$ International Telecommunication Union, "ITU-T P.58. Head and torso simulator for telephonometry," Recommendation, Geneva (1996).

${ }^{5}$ International Telecommunication Union, "ITU-T P.57. Artificial ears," Recommendation, Geneva (2009).

${ }^{6} \mathrm{~K}$. Ueno, K. Kato, and K. Kawai, "Effect of room acoustics on musicians' performance. Part I: Experimental investigation with a conceptual model," Acta. Acust. Acust. 96, 505-515 (2010).

${ }^{7}$ D. Pelegrin-Garcia and J. Brunskog, "Prediction of vocal effort and speakers' comfort in lecture rooms," in Proceedings of Inter-Noise 2009, Ottawa, Canada (2009).

${ }^{8}$ D. Pelegrin-Garcia and J. Brunskog, "Natural variations of vocal effort and comfort in simulated environments," in Proceedings of EAA Euroregio Congress on Sound and Vibration 2010, Ljubljana, Slovenia (2010). 